\title{
Enhancement of Rossby Wave Breaking by Steep Potential Vorticity Gradients in the Winter Stratosphere
}

\author{
R. K. SCOTT \\ Department of Applied Physics and Applied Mathematics, Columbia University, New York, New York \\ D. G. DRITSCHEL \\ School of Mathematics and Statistics, University of St. Andrews, St. Andrews, United Kingdom \\ L. M. Polvani \\ Department of Applied Physics and Applied Mathematics, Columbia University, New York, New York \\ D. W. WAUGH \\ Department of Earth and Planetary Sciences, The Johns Hopkins University, Baltimore, Maryland
}

(Manuscript received 14 February 2003, in final form 4 November 2003)

\begin{abstract}
This work investigates the extent to which potential vorticity gradients affect the vertical propagation of planetary-scale Rossby waves on the edge of a stratospheric polar vortex and their eventual nonlinear saturation and breaking. Using two different numerical modeling approaches, it is shown that wave propagation and wave breaking are significantly reduced when the potential vorticity gradients at the vortex edge are anomalously weak. The efficiency of the first model, based on high-resolution contour dynamics, permits a full exploration of the parameter space of wave forcing amplitude and edge steepness. A more realistic primitive equation model in spherical geometry both confirms the contour dynamics results and highlights some direct implications for stratospheric modeling in more comprehensive models. The results suggest that stratospheric models using horizontal resolutions of spectral T42 or less may significantly underestimate the vertical propagation and breaking of planetary waves, and may consequently misrepresent such important stratospheric processes as the mean meridional circulation, sudden warmings, and the mixing of chemically distinct polar and midlatitude air.
\end{abstract}

\section{Introduction}

An understanding of the dynamics of the polar winter stratosphere is crucial to an understanding of the chemical and thermal structure of the middle atmosphere. The low temperatures inside the stratospheric polar vortex and the isolation of polar air from midlatitudes by strong potential vorticity gradients at the vortex edge are both key factors in determining the extent of ozone depletion in polar regions (e.g., McIntyre 1990; WMO 1999, chapter 7 and references therein). On longer time scales, the strength of the mean meridional circulation determines the transport of radiatively and chemically important species throughout the middle atmosphere. More recently, the possibility that the stratosphere may also play a role in the surface climate has received consid-

Corresponding author address: Richard Scott, Northwest Research Associates, Inc., P.O. Box 3027, Bellevue, WA 98009-3027.

E-mail: scott@nwra.com erable attention (e.g., Hartmann et al. 2000, for a recent discussion).

The dynamical structure of the stratosphere is characterized by the strong potential vorticity (PV) gradients of the winter polar vortex, on which planetary-scale Rossby waves, generated in the troposphere, are able to propagate into the upper stratosphere. Amplification of these waves with height, and their eventual nonlinear saturation and breaking, leads to mixing of polar and midlatitude air and contributes to the driving of the mean meridional circulation (e.g., McIntyre 1992, for an overview). An accurate understanding of the details of wave propagation and breaking is therefore a prerequisite for accurate climate modeling.

Traditionally, it is accepted that strong PV gradients inhibit wave breaking and the mixing and transport of air, acting as a deformable, dynamically elastic barrier (Hoskins et al. 1985). The elasticity associated with strong PV gradients allows large-amplitude reversible deformations to occur without the irreversible mixing 
associated with wave breaking. Thus, the strong PV gradients at the edge of the stratospheric polar vortex act as a barrier to the mixing of polar and midlatitude air, as was illustrated by the two-dimensional simulations of Juckes and McIntyre (1987).

In three dimensions, however, the situation is more complicated. As a wave packet propagates up a steep vortex edge, the presence of large PV gradients (and thus strong PV elasticity) will inhibit wave breaking at lower levels, and most of the (pseudo-)momentum associated with the wave packet will be transferred to high levels. Together with the exponential amplification with height due to the density decrease, enhanced upward wave propagation on steep PV gradients might thus lead to enhanced wave breaking and mixing in the upper stratosphere.

This possibility was suggested recently by Polvani and Saravanan (2000), who found weaker wave propagation on a broader vortex edge in a primitive equation model. However, since their study used relatively low resolution (spectral T42), even their steep edge was necessarily already much broader than that likely to exist in the winter stratosphere. Further, as they only considered a single case, it is not clear to what extent the stronger PV elasiticity present in the stratosphere will lead to enhanced vertical propagation. This is the issue addressed in the present paper.

Because of the dependence of wave propagation on PV gradients, a natural question is how steep are the actual gradients in the winter stratosphere. Aircraft observations (e.g., Tuck 1989; Plumb et al. 1994; Waugh et al. 1994) reveal jumps in the concentration of chemical species from interior to exterior vortex values occurring over length scales of the order of $100 \mathrm{~km}$ or less, suggesting that the width of the vortex edge is similarly confined in latitude. Theoretical arguments and idealized numerical modeling (Legras and Dritschel 1993; Legras et al. 2001) also indicate the development of high-vorticity gradients from smoother initial distributions in the presence of a background shear or strain flow: the erosion or stripping of low-vorticity air at the outer edge of the vortex leads to an intensification of vorticity gradients.

In this paper we make a detailed study of the effect of the edge width on wave propagation using two different models. The first of these is the Contour-Advective Semi-Lagrangian (CASL) model introduced by Dritschel and Ambaum (1997). In the configuration used in this study, this is a fully three-dimensional model with variable Coriolis parameter, and therefore captures many important features of the stratospheric circulation. On the other hand, the high efficiency of this method allows for a detailed exploration of a two-dimensional parameter space comprising the wave forcing amplitude and vortex edge width.

The second model we use is a primitive equation model in spherical geometry, identical to the dynamical cores of many global circulation models (GCMs). This model confirms the robustness of the CASL model results and highlights some implications for stratospheric modeling in more comprehensive models. Our results indicate that upward wave propagation and wave breaking in the upper vortex is, in general, significantly weaker when the vortex edge PV gradients are smooth than when they are steep. Since typical numerical models, particularly climate models, cannot resolve the steep PV gradients indicated by observations, these results have important implications for stratospheric modeling.

The paper is organized as follows. In section 2 , we outline the new features of the CASL model that enable a more realistic representation of the polar stratosphere, and describe the construction of a vortex with a controlled, variable edge width. Section 3 provides an illustration of the effect of the edge width on the wave propagation in the simplest setting, that of linear wave propagation on an $f$ plane. In section 4 , we extend the analysis to the more realistic setting of nonlinear waves on a polar $\gamma$ plane, the polar equivalent to the midlatitude $\beta$ plane. In section 5 , we present the results of the primitive equation model. Implications for low-resolution stratospheric climate modeling are discussed in section 6.

\section{CASL model details, forcing, and initial conditions}

\section{a. Model domain and equations}

The CASL model solves the quasigeostrophic equations in a cylindrical domain using a polar coordinate system $(r, \theta, z)$ that rotates about the cylindrical axis $r=0$ at the rate $\Omega=f_{0} / 2$ (Macaskill et al. 2003). Isothermal boundary conditions are imposed at $z=0$, $D$. In this coordinate system the equations take the form

$$
\begin{gathered}
\frac{D q}{D t} \equiv \frac{\partial q}{\partial t}+u \frac{\partial q}{\partial r}+\frac{v}{r} \frac{\partial q}{\partial \theta}=0, \\
\nabla_{h}^{2} \psi+\frac{1}{\rho_{0}} \frac{\partial}{\partial z}\left(\rho_{0} \frac{f_{0}^{2}}{N_{0}^{2}} \frac{\partial \psi}{\partial z}\right)=q-f(r), \\
\frac{\partial \psi}{\partial z}=0 \quad \text { at } z=0 \text { and } z=D, \\
(u, v)=\left(-\frac{1}{r} \frac{\partial \psi}{\partial \theta}, \frac{\partial \psi}{\partial r}\right) .
\end{gathered}
$$

Here, $q(r, \theta, z, t)$ is the PV, $\psi$ is the geostrophic streamfunction, $(u, v)$ are the radial and azimuthal components of the geostrophic velocity, $\rho_{0}$ is the density, $D$ is the domain height, $f(r)$ is the Coriolis parameter, and $N_{0}$ is the buoyancy frequency. Throughout this paper we consider the situation, relevant to the stratosphere, where $N_{0}=0.0214 \mathrm{~s}^{-1}$, and $\rho_{0}=\rho_{s} e^{-z / H}$, for some constant $\rho_{s}$ and constant scale height $H=6146 \mathrm{~m}$, chosen so 
that the vertical domain corresponds to the region of the atmosphere lying between $12-$ and $48-\mathrm{km}$ altitude.

The use of a cylindrical domain offers two main advantages over the doubly periodic domain used in a previous studies. First, the axisymmetry of the domain allows an easy implementation of a stretched grid, where grid points are concentrated near the pole, the region of interest, and a large domain can be used to distance unwanted boundary effects. The radial grid used in the present study has grid points uniformly spaced in $r^{1 / 2}$, with a domain size of $r=30 L_{R}$, where $L_{R}=902 \mathrm{~km}$ is the Rossby deformation radius.

The second, and more significant, advantage of the cylindrical domain lies in the ability to represent a variable Coriolis parameter. By choosing $f=f_{0}-\gamma r^{2}$, we obtain the first-order approximation to the actual variation of the earth's Coriolis parameter near the pole, the so-called $\gamma$-plane approximation. This is the polar equivalent to the midlatitude $\beta$ plane. The inclusion of a variable $f$ constitutes an important advance over the $f$-plane approximation in the applicability of this system to polar stratospheric flows, in that Rossby waves are now able to propagate away from the polar vortex on the background PV gradient. In a single-layer barotropic system, Waugh (1993) used this procedure to represent a variable $f$ on an infinite plane and found good agreement between the variable $f$, planar results, and those obtained with full spherical geometry. The $f$-plane approximation is recovered by setting $f(r)=f_{0}$ in (1).

Note that the radial and azimuthal coordinates in the cylindrical domain can be identified with the latitudinal and zonal coordinates in spherical geometry near the pole by the correspondence $(r, \theta) \leftrightarrow(a \cos \phi, \lambda)$, where $\phi$ is latitude, $\lambda$ is longitude, and $a$ is the earth's radius. The axial coordinate $z$ in the cylindrical domain corresponds to the ordinary height.

The Eqs. (1) are discretized following Dritschel and Saravanan (1994) and Waugh and Dritschel (1999). The vertical domain is divided up into $L=30$ layers of uniform depth. In each layer, the PV distribution $q(r, \theta, z, t)$ is assumed to be piecewise uniform, whereby a continuous distribution is represented by a series of small discontinuities across PV contours. Note that in this study, unlike that of Waugh and Dritschel (1999), the use of many contours in each horizontal layer permits high resolution of a fully three-dimensional PV distribution. The horizontal grid used for the PV inversion in (1b) comprises 96 radial and 192 azimuthal grid points; the PV itself is retained on a grid 4 times finer.

\section{b. Initial conditions}

We construct a set of axisymmetric, barotropic vortices centered on the "pole" at $r=0$ in such a way that the width of the vortex edge depends on a single external dimensionless parameter $\varepsilon$. The construction is such that the fundamental properties of the vortices are identical for different edge widths. In particular, we require the anomalous circulation and the angular impulse of the vortex, as well as the location of the zero wind line in the velocity field outside the vortex to be all independent of $\varepsilon$.

On the $f$ plane, the initial PV distribution is independent of height $z$ and takes the following form:

$$
q(r)= \begin{cases}q_{i} & r<r_{1}(\varepsilon) \\ q_{i}-\left(q_{i}-q_{o}\right) \frac{r^{2}-r_{1}^{2}}{r_{2}^{2}-r_{1}^{2}} & r_{1}(\varepsilon)<r<r_{2}(\varepsilon) \\ q_{o} & r>r_{2}(\varepsilon),\end{cases}
$$

where $q_{i}$ is the PV at the vortex center, inside the inner edge at $r=r_{1}, q_{o}$ is the exterior PV, outside the outer edge at $r=r_{2}$, and the edges are defined by $r_{1}^{2}=R^{2}(1$ $-\varepsilon$ ) and $r_{2}^{2}=R^{2}(1+\varepsilon)$, where $R$ is the mean vortex radius.

The special case of $\varepsilon=0$ corresponds to an infinitely steep edge, that is, a single step with $r_{1}=r_{2}=R$. In this case, values of $q_{i}$ and $q_{o}$ are chosen to match the vortex considered by Dritschel and Saravanan (1994): $q_{i}=1.3 f_{0}, q_{o}=0.9 f_{0}$, and $R=3 L_{R}$. This profile has a zero wind line at $r_{0}=6 L_{R}$ and easterlies for $r>$ $r_{0}$. The peak winds occur at $r=R$, where $u=$ $(1 / 2)\left(q_{i}-f_{0}\right) R \approx 59 \mathrm{~m} \mathrm{~s}^{-1}$ in dimensional units. For $\varepsilon>0$ we choose $q_{i}(\varepsilon)$ and $R(\varepsilon)$ such that the anomalous circulation, the angular impulse and zero wind line are the same as for $\varepsilon=0$.

From the definition of the anomalous circulation of the (barotropic) vortex

$$
\kappa=\int_{0}^{r_{2}}\left(q-q_{o}\right) r d r=\frac{1}{2}\left(q_{i}-q_{o}\right) R^{2},
$$

the condition that determines the location $r_{0}$ of the zero wind line can be expressed as

$$
\frac{\kappa}{r_{0}}=\frac{1}{2}\left(f_{0}-q_{o}\right) r_{0} .
$$

Thus, the requirement that $\kappa$ and $r_{0}$ be fixed implies that $q_{o}$ is the same for all $\varepsilon$ (with $q_{o}=0.9 f_{0}$ ). Finally, the angular impulse is given by

$$
J=\int_{0}^{r_{2}}\left(q-q_{o}\right) r^{3} d r=\frac{1}{4}\left(q_{i}-q_{o}\right)\left(1+\frac{1}{3} \varepsilon^{2}\right) R^{4} .
$$

Requiring $\kappa$ and $J$ to be independent of $\varepsilon$ allows (3) and (5) to determine $R$ and $q_{i} / f_{0}$ for each value of $\varepsilon$. Values of $R$ and $q_{i}$ for $\varepsilon=0,0.1, \ldots, 1.0$ are shown in Table 1, while the initial PV profile $q$ given by (2) and the corresponding velocity profile are shown in Fig. 1a and Fig. 1c, respectively.

For the $\gamma$-plane case, we use the relative vorticity profile as in the $f$-plane case, which means that the PV profile $q$ is modified from (2) by the addition everywhere of a term $-\gamma r^{2}$. This construction ensures that 
TABLE 1 . Values of mean vortex radius $R$ and interior vorticity anomaly $q_{i} / f_{0}$ for different values of the edge width parameter $\varepsilon$.

\begin{tabular}{ccc}
\hline \hline$\varepsilon$ & $R$ & $q_{i} / f_{0}$ \\
\hline 0.0 & 3.0000000 & 1.3000000 \\
0.1 & 2.9950125 & 1.3013333 \\
0.2 & 2.9801978 & 1.3053333 \\
0.3 & 2.9559878 & 1.3120000 \\
0.4 & 2.9230641 & 1.3213333 \\
0.5 & 2.8823068 & 1.3333333 \\
0.6 & 2.8347335 & 1.3480000 \\
0.7 & 2.7814366 & 1.3653333 \\
0.8 & 2.7235239 & 1.3853333 \\
0.9 & 2.6620695 & 1.4080000 \\
1.0 & 2.5980762 & 1.4333333 \\
\hline
\end{tabular}

the anomalous circulation and angular impulse due to the vortex are identical to those in the $f$-plane case, since the anomalous PV $q-q_{o}$ appearing in the integrands in (3) and (5) is now $q-\left(q_{o}-\gamma r^{2}\right)$. Similarly, the velocity profile associated with this PV profile is identical to that in the $f$-plane case since the background vorticity $f$ appearing in (1b) is now given by $f_{0}-\gamma r^{2}$, and the $\gamma$ contributions from $q$ and $f$ cancel. The values of $R(\varepsilon)$ and $q_{i}(\varepsilon)$ are therefore identical to those obtained with the $f$ plane and given in Table 1 . The PV profile in the $\gamma$-plane case is shown in Fig. 1b.

\section{c. Topographic forcing}

Following Dritschel and Saravanan (1994), waves are forced in the model using a fixed lower boundary distribution of PV to represent the flow over bottom topography $\eta(r, \theta)$. This is achieved by adding a contribution $q_{b}$ to the total PV distribution, where $q_{b}$ is given by

$$
q_{b}=-a \frac{f_{0}}{D} \eta(r, \theta) \delta(z) T(t),
$$

with $a$ a nondimensional forcing amplitude, $\delta(z)$ the Dirac delta function, and $T(t)$ a time-dependent ramp function. The form of $\eta$ is an azimuthal (or zonal) wavenumber-1 bump with a radial maximum at $r=r_{m}=$ $3 L_{R}$, near the vortex edge,

$$
\eta(r, \theta)= \begin{cases}\sin ^{2} \frac{\pi r}{2 r_{m}} \cos \theta & r \leq 2 r_{m} \\ 0 & r>2 r_{m},\end{cases}
$$

while the time dependence is a single pulse with maximum at $t=t_{m}=5$ days and zero after $t=10$ days,

$$
T(t)= \begin{cases}\sin ^{2} \frac{\pi t}{2 t_{m}} & t \leq 2 t_{m} \\ 0 & t>2 t_{m} .\end{cases}
$$

As argued in Polvani and Saravanan (2000), a pulsed forcing is a better representation than steady forcing of the time dependence typically observed in the tropopause geopotential height field before major strato-
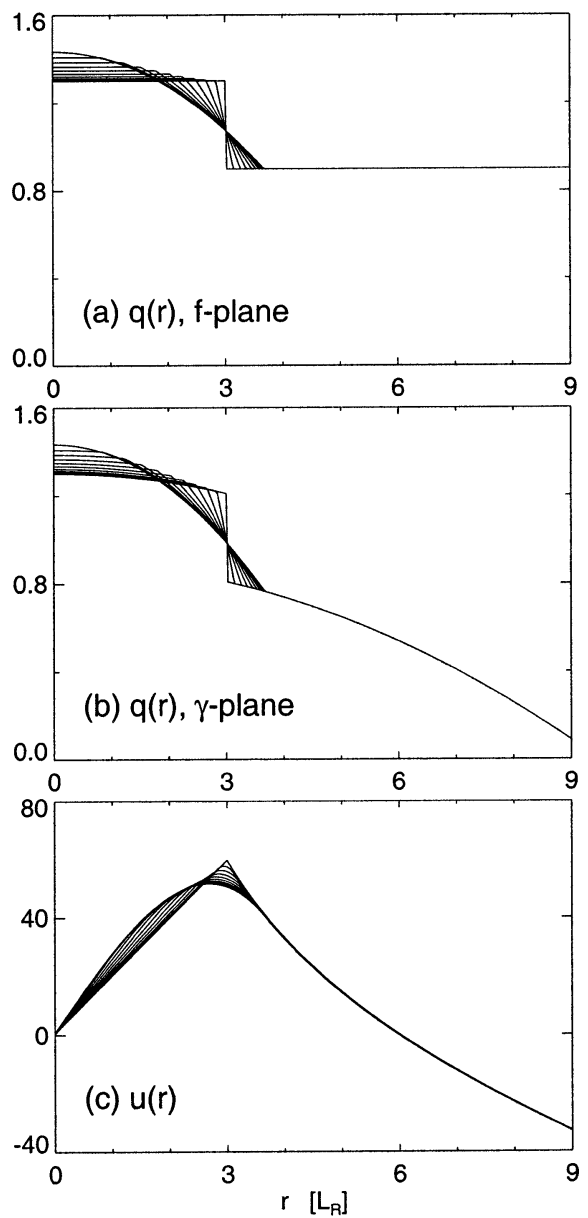

FIG. 1. (a), (b) Potential vorticity $q$ (in $f_{0}$ ), and (c) zonal velocity $u$ (in $\mathrm{m} \mathrm{s}^{-1}$ ) of the initial condition for varying edge width $\varepsilon$, as a function of radial coordinate $r$ (in $L_{R}=902 \mathrm{~km}$ ). The $f$-plane case is shown in (a) and the $\gamma$-plane case in (b). The zonal velocity is the same in both cases.

spheric warming events. Further, because we are interested in the wave propagation characteristics of a carefully controlled vortex edge, it turns out that pulsed forcing has an additional advantage in that vortex destruction is confined to the top of the domain and the lower vortex edge remains relatively unaffected by the wave forcing. As will be seen below, this enables us to consider more cleanly the effect of the vortex edge width on the upper vortex dynamics.

\section{3. $f$-plane results}

To illustrate the effect of the edge width on wave propagation in the simplest possible setting, we present results of quasi-linear dynamics on the $f$ plane. Rather than perform a full linear analysis of this system, it is easier to simply force the model with a very weak forcing amplitude, such that the dynamics remains linear over the time interval of interest. For each member of the set of vortices described by (2) with $\varepsilon=0,0.1, \ldots, 1.0$, that 


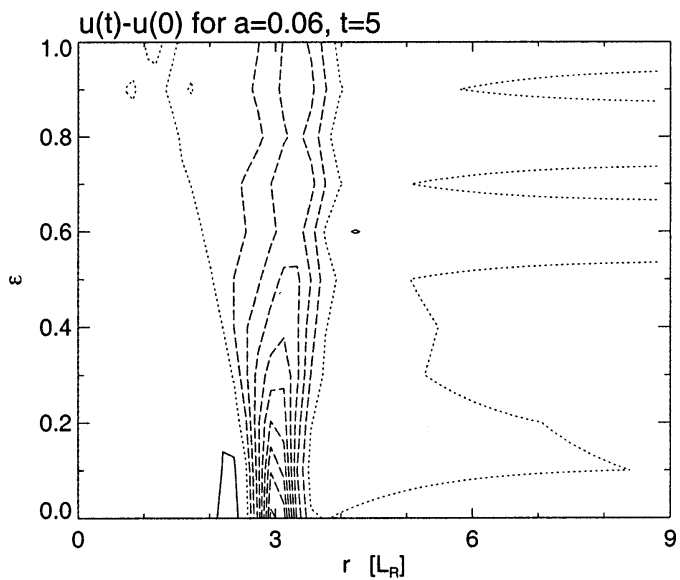

FIG. 2. Deceleration of the upper vortex in the $f$-plane case, defined as $\bar{u}(t)-\bar{u}(0)$ at time $t=5$ days, averaged over the top $20 \%$ of the vortex, as a function of radius and $\varepsilon$. Topographic forcing amplitude is $a=0.06$. The zonal averages are taken with respect to the global centroid of the vortex. The contour interval is $0.5 \mathrm{~m} \mathrm{~s}^{-1}$; positive contours are solid, negative contours are dashed, and the zero contour is dotted.

is, the 11 profiles depicted in Figs. 1a,c for initial conditions, the Eqs. (1) are integrated for 30 days, with wave forcing at the lower boundary using (6) with amplitude $a=0.06$ (corresponding to about $184 \mathrm{~m}$ ).

A convenient measure of the effect of the waves on the vortex is the deceleration of the upper $20 \%$ of the vortex, defined by $\bar{u}(t)-\bar{u}(0)$, where the overbar denotes a zonal average with respect to the global centroid (mass weighted) of the entire vortex. Taking the zonal average with respect to the vortex centroid is important here, since the $f$ plane has no preferred pole, and a large part of the vortex response to the wave forcing is manifested by the simple translation of the vortex with respect to the origin $r=0$. Since this translation is greatly inhibited by the planetary vorticity in the atmosphere and in the $\gamma$-plane cases below, it is natural to restrict attention here to centroid-relative quantities, thereby considering only those interactions that distort the shape of the vortex.

Figure 2 shows the upper vortex deceleration $\bar{u}(t)-$ $\bar{u}(0)$ at $t=5$ days as a function of radial coordinate (equivalent to the colatitude in spherical geometry) and the external parameter $\varepsilon$ governing the vortex edge width. There is a clear decrease in the deceleration at the vortex edge as $\varepsilon$ is increased from 0 (steep edge) to 1 (broad edge). Values of deceleration for $\varepsilon \geq 0.6$ are less than a third those for $\varepsilon=0$.

Note that the confinement of the deceleration to the vortex edge is due to the fact that waves cannot propagate away from the edge region on the $f$ plane, as this is the only region in which the PV gradient is nonzero. Thus, the strong difference in the response for different $\varepsilon$ is due completely to the ability of waves to propagate on the PV gradients of the vortex edge.

Using the same zonal averaging with respect to the vortex centroid, the Eliassen-Palm (EP) flux $\mathbf{F}$, and EP flux convergence $-\boldsymbol{\nabla} \cdot \mathbf{F}$ (e.g., Andrews et al. 1987, chapter 3) also show decreased wave propagation and flux convergence on a broad vortex edge, as can be seen in Figs. 3a,b for the cases $\varepsilon=0.1$ and $\varepsilon=0.7$ at $t=$ 5 days. In the upper vortex, the EP flux divergence for $\varepsilon=0.7$ is less than a half that for $\varepsilon=0.1$. We observe that the latitudinally integrated upward EP fluxes are similar in each case (not shown, but see the difference plotted in Fig. 3c). Thus, the differences in the EP flux convergence arise primarily from the difference in the distribution of wave flux on the vortex edges.

At low levels, the flux arrows for $\varepsilon=0.1$ are more vertically aligned, while those for $\varepsilon=0.7$ deviate more toward low latitudes, that is, toward the outer region of (a) $\varepsilon=0.1$

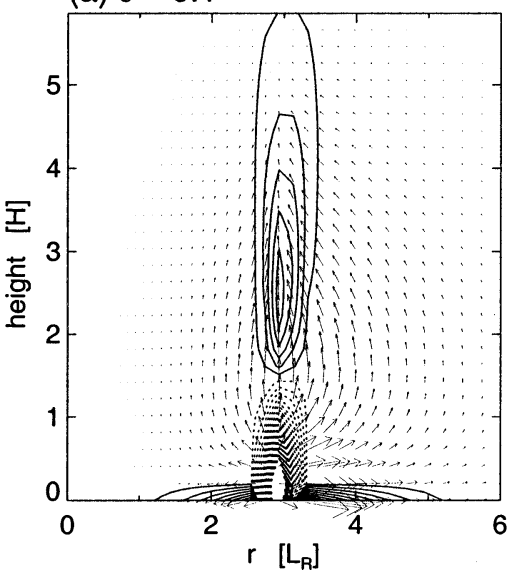

(b) $\varepsilon=0.7$

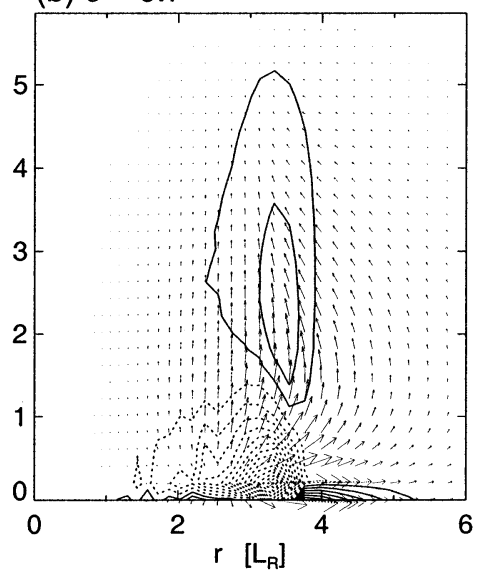

(c) difference

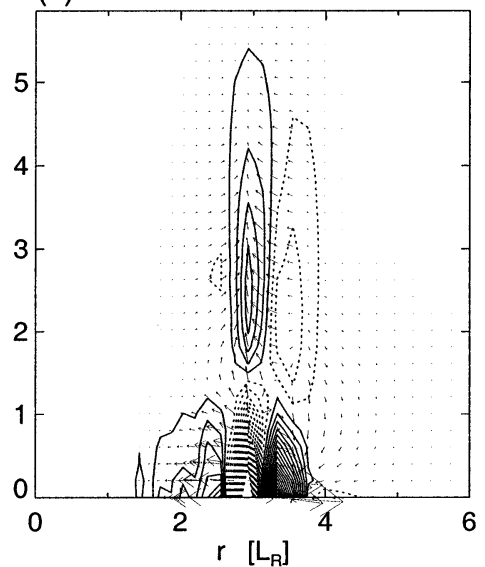

FIG. 3. EP flux and EP flux convergence for the $f$-plane case for (a) $\varepsilon=0.1$, (b) $\varepsilon=0.7$, and (c) the difference, on day 5 . Topographic forcing amplitude is $a=0.06$. The zonal averages are taken with respect to the global centroid of the vortex. Graphical conventions follow Dunkerton et al. (1981) with appropriate modifications for cylindrical geometry. The arrow lengths in (c) have been magnified $\times 5$ with respect to those in (a) and (b). 
(a) $\varepsilon=0.1$

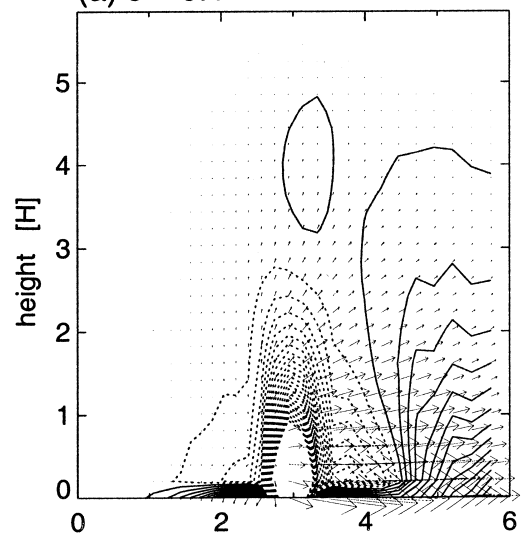

(d) $\varepsilon=0.1$

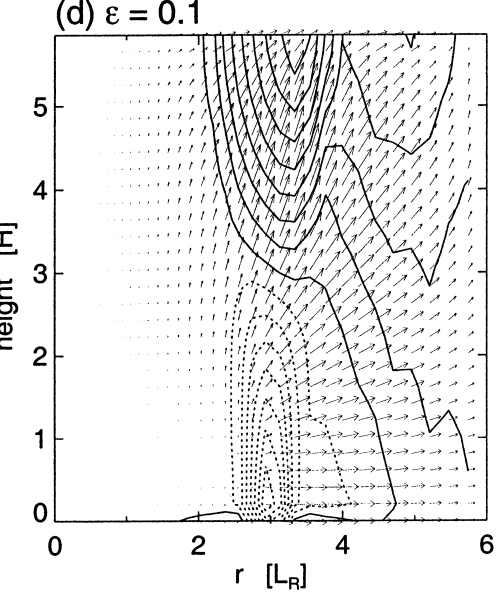

(b) $\varepsilon=0.7$

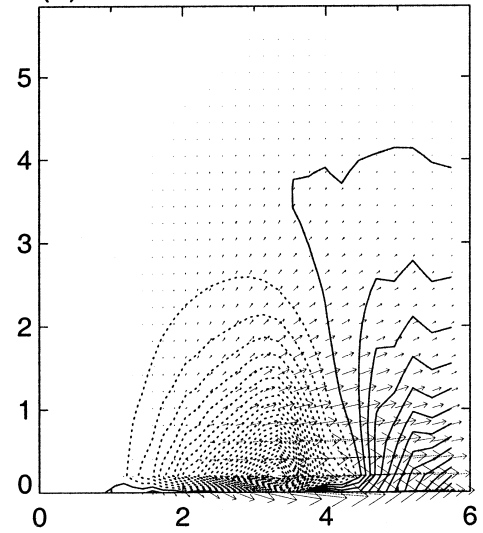

(e) $\varepsilon=0.7$

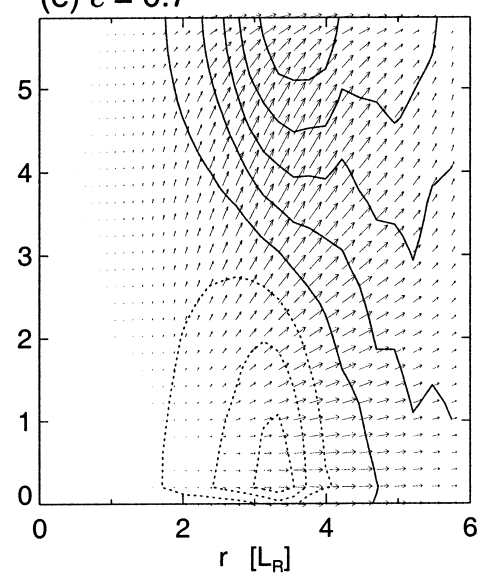

(c) difference

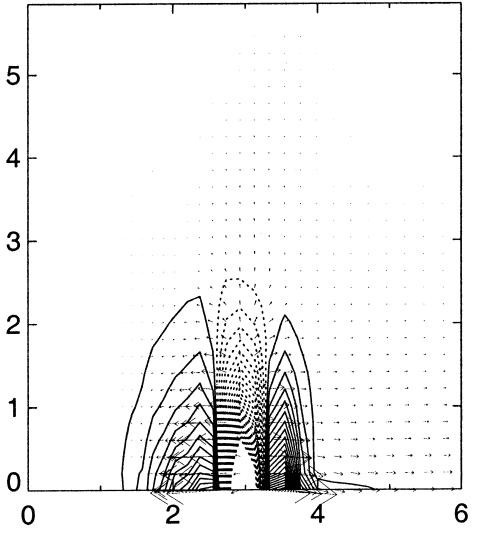

(f) difference

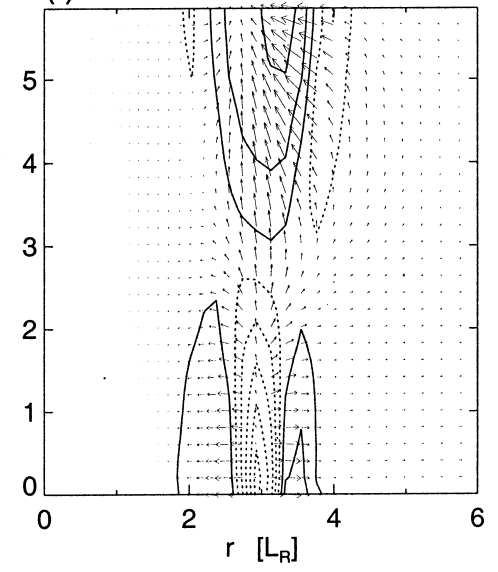

FIG. 4. The EP flux and EP flux convergence for the $\gamma$-plane case for (a) $\varepsilon=0.1$, (b) $\varepsilon=0.7$, and (c) the difference, on day 5. (d)-(f) As in (a)-(c), but for the density-weighted EP flux and EP flux convergence (i.e., the force per unit mass $\rho^{-1} \boldsymbol{\nabla} \cdot \mathbf{F}$ ). Topographic forcing amplitude is $a=0.06$. The zonal averages are taken with respect to the pole. The arrow lengths in (c) and (f) have been magnified $\times 5$ with respect to those in (a), (b), (d), and (e).

the vortex edge. The net result at upper levels is a weaker region of flux convergence for $\varepsilon=0.7$ and this region is shifted toward the outer edge. This pattern can be seen most clearly in the difference plot in Fig. 3c (note that the arrows have been magnified by a factor of five). The low-latitude shift of EP flux divergence explains the feature seen in Fig. 2 that the deceleration at larger $\varepsilon$ lies on the outer part of the vortex edge.

\section{4. $\gamma$-plane results}

The linear $f$ plane is useful for the purposes of illustrating the direct effect of the edge width on the wave propagation. However, waves cannot propagate away from the vortex edge in that system and this represents a serious discrepancy from the behavior of the winter stratosphere. The $\gamma$-plane approximation presented in this section, with a variable Coriolis parameter, is an important improvement that allows waves to propagate away from the vortex edge on the background PV gradient.
Note that, on the $\gamma$ plane, the origin $(r=0)$ can be naturally identified with the pole in spherical geometry, and so it is more natural here to take zonal averages with respect to $r=0$ than to the vortex centroid. In fact, because the background PV of the $\gamma$ plane acts to oppose motion of the vortex from the origin, the differences between the two zonal averages are less marked than for the $f$-plane case.

\section{a. Linear regime}

We first show that the reduction in wave propagation on the broad edge vortex, illustrated for the $f$-plane case above, is robust to the inclusion of the variable Coriolis parameter of the $\gamma$ plane. Since waves can now propagate away from the vortex edge to low latitudes on the background potential vorticity gradient, details of the vortex edge may be less important than before.

Figures $4 \mathrm{a}-\mathrm{c}$ show the EP flux and flux convergence at $t=5$ days for a weak forcing amplitude $a=0.06$, that is, the $\gamma$-plane case equivalent of Fig. 3. The EP 
flux is now clearly dominated by a strong horizontal component indicating wave propagation to low latitudes, and there is strong wave flux convergence in the region of weak winds near $r=6 L_{R}$. Horizontal propagation is here much stronger than vertical propagation up the vortex edge. Further, both cases $\varepsilon=0.1$ and $\varepsilon$ $=0.7$ show similar patterns of horizontal propagation and flux convergence outside the vortex edge.

To see the differences in the vertical component of the EP flux between low and high $\varepsilon$ it is convenient to consider the density-weighted EP flux $\rho^{-1} \mathbf{F}$ and flux convergence $\rho^{-1} \boldsymbol{\nabla} \cdot \mathbf{F}$ to emphasize the upper part of the domain (Figs. 4d-f). Now, a clear reduction can be seen in the upper-level wave flux convergence obtained with the broad edge, although the difference is slightly less than that observed on the $f$ plane. Note that, as in the lower part of the domain, there is a similar magnitude of horizontal flux to low latitudes in both the steep and the broad edge cases. Thus, the effect of the broad edge is not simply to allow more wave flux to escape the vortex edge to low latitudes. The difference plot shown in Fig. 4f highlights this: the main difference is in the upward propagation on the vortex edge, rather than the horizontal propagation to low latitudes.

Finally, we remark that the upper vortex deceleration shows a similar dependence on $\varepsilon$ as that found in the $f$-plane case above (not shown for the forcing amplitude $a=0.06$ used above, but see Fig. 6a below for the deceleration in a similar quasi-linear regime).

\section{b. Nonlinear regime}

As a more realistic representation of the stratospheric evolution we turn now to the fully nonlinear response to larger amplitude wave forcing. Figure 5 illustrates the dependence of the upper vortex evolution on a range of forcing amplitudes $a=0.10, a=0.18$, and $a=0.26$ for a given edge width, $\varepsilon=0.4$. These forcing values have been selected as representative of different intensities of wave breaking. At small amplitude $(a=0.10$, or about $307 \mathrm{~m}$ ) a very thin filament of material from the outer edge is stripped off the vortex; at intermediate amplitude $(a=0.18$, or about $553 \mathrm{~m})$ material from the whole edge region is stripped off; and at large amplitude ( $a=0.26$, or about $799 \mathrm{~m}$ ) the whole vortex is violently distorted.

Two features of Fig. 5 are worth noting. First, the dashed lines indicate the contours used to represent the $\gamma$-plane effect. These contours represent dynamically active PV, just like the contours used to represent the vortex edge (solid in the figure). A criticism of contour dynamics methods in the past has frequently been the inability to represent a continuous PV distribution. The distribution of contours in the figure, however, illustrates that the model used in this configuration is capable of representing a complex layerwise two-dimensional flow field and a planetary vorticity gradient at high resolution.
The second important feature of Fig. 5 is the formation of steep gradients at the vortex edge, most clearly seen for $a=0.18$ and $a=0.26$. This is an illustration of the vortex stripping and gradient intensification discussed by Legras and Dritschel (1993), and an indication that the stratospheric polar vortex edge may be significantly steeper than can be adequately resolved in traditional psuedo-spectral or gridpoint models at low to moderate resolutions.

For each of the cases shown in Fig. 5 we consider next the dependence of the wave breaking on the vortex edge thickness parameter $\varepsilon$. As an illustration of the dependence on $\varepsilon$ in the linear regime, we consider the case $a=0.10$ at early times, before the onset of wave breaking. The deceleration of the upper vortex in this case at $t=4$ days is shown in Fig. 6a, again as a function of radius (latitude) and $\varepsilon$. As in the case of the $f$ plane, the deceleration on the broad vortex edge $(\varepsilon \geq 0.8)$ is around a third of that on the steep vortex edge $(\varepsilon=0)$, and is concentrated toward the outer edge region. Thus, as far as the linear dynamics of the upper vortex is concerned, the addition of the $\gamma$ plane has little effect on the main result, even although most of the wave activity propagates away from the vortex to low latitudes at lower levels. As before, it is the PV gradients on the vortex edge that determine how much wave activity can propagate into the upper levels of the domain.

Figures $6 \mathrm{~b}, \mathrm{c}$ shows the same quantity, $\bar{u}(t)-\bar{u}(0)$, but for $a=0.18$ at $t=14$ days and $a=0.26$ at $t=$ 22 days. In these cases, because of substantial filamentation of the vortex, there is a large deceleration in the surf zone region outside the vortex edge, around $4-5 L_{R}$ from the pole. Although this deceleration appears relatively insensitive to $\varepsilon$, it is clear from the figures that the deceleration at the vortex edge itself, around $3 L_{R}$, again shows a strong dependence on $\varepsilon$, with values varying by around a factor of two between steep and broad edge cases. In general, the width of the region that undergoes strong deceleration is reduced as $\varepsilon$ is increased.

To examine in more detail the nonlinear dynamics of the cases with stronger wave forcing, we show the PV in the uppermost layer of the domain for different values of $\varepsilon$. Figure 7 illustrates the case $a=0.18$ for edge widths $\varepsilon=0.1, \varepsilon=0.4$, and $\varepsilon=0.7$. Since the initial vortex in each case has the same basic properties (circulation and angular impulse) the evolution of the wave breaking is very similar in each case. An important difference, however, is seen in the inner edge contours, which break for $\varepsilon=0.1$ but remain intact for $\varepsilon=0.7$. This can be understood kinematically from the proximity of the contours to the stagnation point set up in flow by the forcing (Polvani and Plumb 1992). It means, however, that, whereas for $\varepsilon=0.1$ polar air from the vortex core is mixed out to low latitudes, for $\varepsilon=0.7$ the vortex core remains isolated from low latitudes. Since a low-resolution pseudo-spectral model may only be capable of representing the edge widths resembling the case $\varepsilon=0.4$ or even $\varepsilon=0.7$, these will clearly 

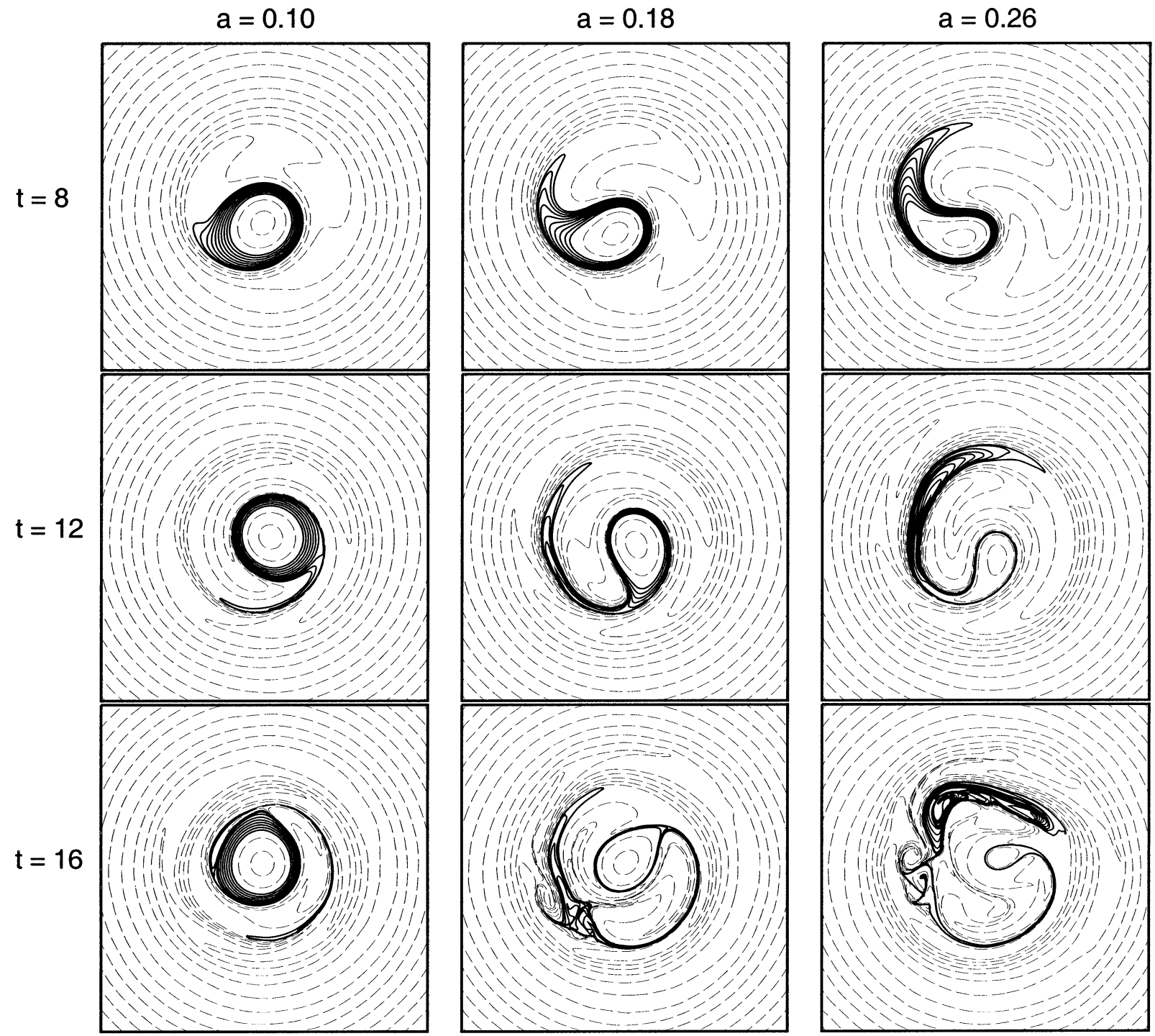

FIG. 5. Potential vorticity of the top model layer, for forcing amplitudes $a=0.10, a=0.18, a=0.26$, at times $t=8, t=12, t=16$ days, respectively, for fixed edge width $\varepsilon=0.4$. The contours representing the vortex edge are solid and those representing the background PV gradient (the $\gamma$ contours) are dashed. The domain of view is the box $|x|,|y| \leq 12 L_{R}$ (the full domain extends out to $30 L_{R}$ ).

underestimate chemical mixing due to wave breaking on the vortex edge.

Figure 8 shows the top layer of the vortex for the strongly forced case $a=0.26$, again for edge widths $\varepsilon$ $=0.1, \varepsilon=0.4$, and $\varepsilon=0.7$. In all cases, the strong wave breaking at $t=14$ days almost completely destroys the vortex in this layer, and air from the vortex core is mixed out into lower latitudes. A careful comparison, however, reveals that the area of the original vortex left behind after this wave breaking event is larger for $\varepsilon=0.7$ than for $\varepsilon=0.1$. This has important consequences for the subsequent vortex evolution. In particular the larger vortex remnant for $\varepsilon=0.7$ has a strong enough circulation to reincorporate much of the ejected filamentary vortex air in vortex merger-type events, which acts to intensify the original vortex. Con- sequently, at $t=22$ days we see that there is a large vortex sitting over the pole for $\varepsilon=0.7$, much of which is reincorporated filamentary vorticity, whereas no such coherent vortex can be seen for $\varepsilon=0.1$.

Finally, we describe briefly the general dependence of wave breaking and changes to the vortex on the full range of the parameters $a$ and $\varepsilon$. To do so, we consider two diagnostics based on a single PV contour taken from the middle of the vortex edge.

The first diagnostic is the wave activity, defined as the deviation from circular of a given midedge contour (see Dritschel and Saravanan 1994, appendix B, for details). The restriction here to a midedge contour is important to remove the large contribution to the wave activity from the filamentary stripping of the outer vortex edge. As the edge width is increased, this stripping 

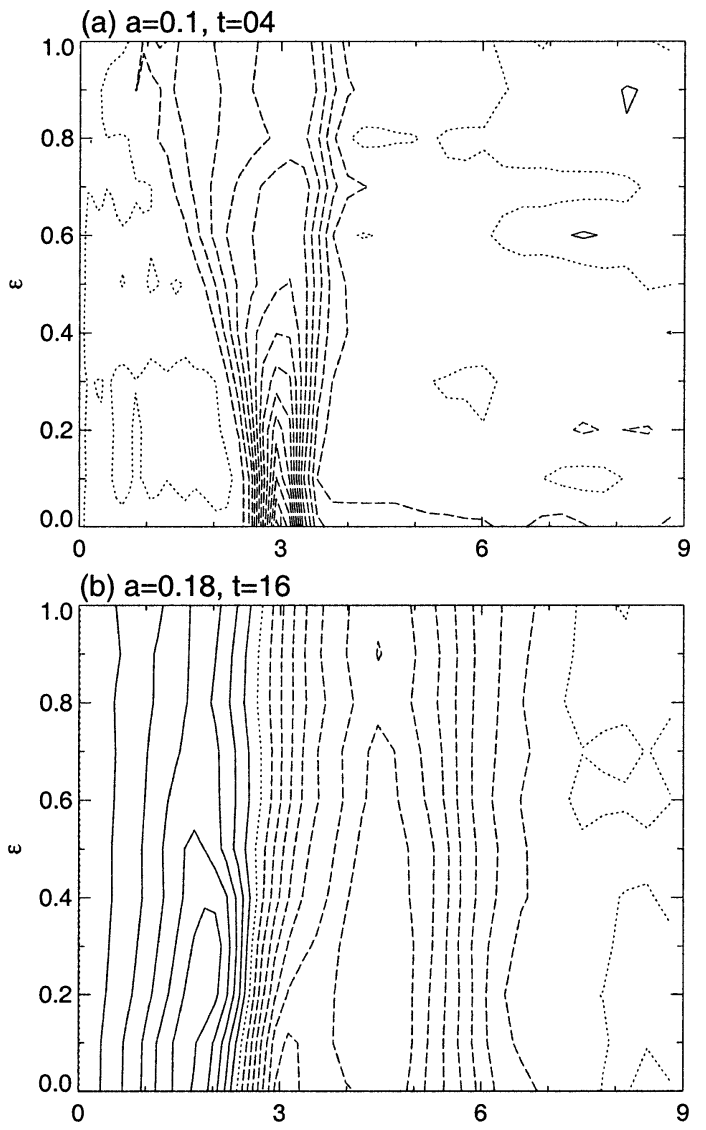

(c) $a=0.26, t=22$

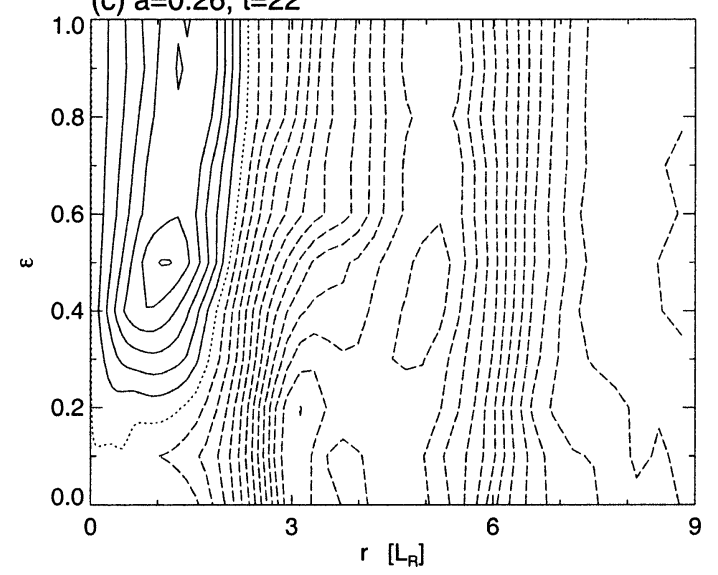

FIG. 6. Deceleration of the upper vortex in the $\gamma$-plane case, defined as $\bar{u}(t)-\bar{u}(0)$, averaged over the top $20 \%$ of the vortex, as a function of radius and $\varepsilon$ : (a) $a=0.10$ at $t=4$ days, (b) $a=0.18$ at $t=16$ days, and (c) $a=0.26$ at $t=22$ days. The contour interval is 0.5 $\mathrm{m} \mathrm{s}^{-1}$ in (a) and $2.5 \mathrm{~m} \mathrm{~s}^{-1}$ in (b) and (c); line styles are as in Fig. 2.

becomes more and more intense (Legras and Dritschel 1993), to the extent that it dominates the total wave activity budget, even though the main vortex core may be relatively undisturbed. We therefore focus on the midedge, defined by the mean PV of the vortex edge, as a suitably representative edge contour.

Figure 9a shows the (logarithm of the) total wave activity based on the midedge contour (contour 6) vertically averaged over the upper $20 \%$ of the vortex and time averaged from $t=10$ to $t=20$ days as a function of forcing amplitude $a$ and edge width $\varepsilon$. The band of steep gradients running diagonally from lower left to upper right indicates a transition from low wave activity to high wave activity. This transition essentially marks the onset of vortex filamentation, where large filaments of vortex air contribute strongly to the total wave activity. For a given forcing amplitude $a$, there is higher wave activity on the left (low $\varepsilon$, or steep edge) than on the right (high $\varepsilon$, or broad edge). For example, the case $a=0.18$ described above intersects this transition region: as can be seen from Fig. 7 , there is strong filamentation of the midedge contour for $\varepsilon=0.1$ but none for $\varepsilon=0.7$. Equivalently, the onset of filamentation on a broader-edged vortex occurs at larger forcing amplitudes $a$ than that on a steep-edged vortex, while at larger $a$ the onset of filamentation occurs for larger $\varepsilon$.

Above the transition region marking the sudden increase in filamentation, further increase in wave forcing and the corresponding increase in vortex destruction does not contribute greatly to a further increase in wave activity. At this point the filamentation has significantly reduced the area and angular impulse of the vortex and so more violent vortex destruction cannot significantly increase the wave activity (a measure of angular impulse and circulation). As an indication of total vortex destruction, therefore, a more useful quantity is the volume of the upper $20 \%$ of the vortex. This is again defined as the volume enclosed by the midedge vortex contour, which has now been suitably coarse grained (e.g., Waugh 1992; Waugh and Plumb 1994; Polvani et al. $1995)$ to remove filamentary debris. Its time average over days 10-20 and is shown in Fig. 9b as a function of $a$ and $\varepsilon$. This shows the volume decreasing uniformly as the forcing amplitude is increased. Again the dependence on $\varepsilon$ is such that a broad edge vortex requires stronger forcing than a steep edge vortex to achieve the same level of vortex destruction.

\section{c. Vertical structure}

The simulations described above all used a pulsed forcing, with the amplitude peaking at 5 days and returning to zero by 10 days. The result is that a single pulse of waves propagates up the vortex and most wave breaking is confined to the upper vortex (compared with steady forcing in which much of the vortex experiences wave breaking; Polvani and Saravanan 2000). That the lower vortex region is not significantly disturbed here is convenient, because we can better isolate the wave propagation characteristics of a variable vortex edge width.

To illustrate the vertical structure of the vortex, we show in Fig. 10 the equivalent vortex radius for each vortex contour after 30 days (by which time most transients have decayed), again using coarse graining to 

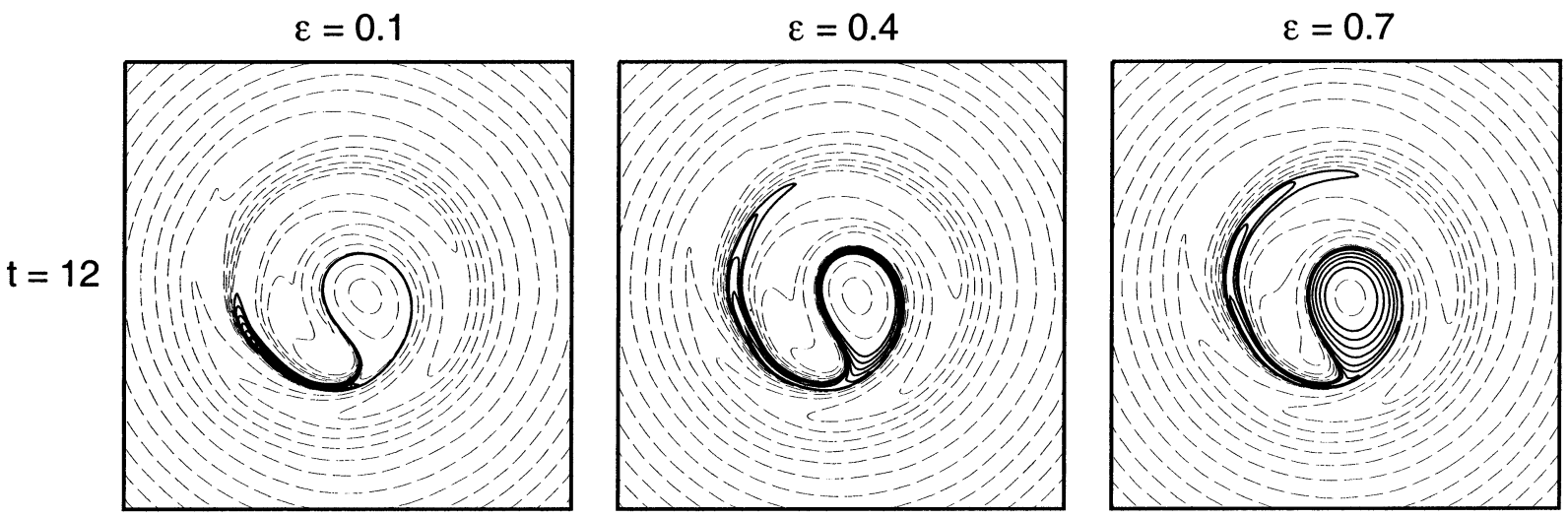

FIG. 7. Potential vorticity of the top model layer, for forcing amplitude $a=0.18$ at time $t=12$ days, for edge width $\varepsilon=0.1, \varepsilon=0.4$ and $\varepsilon=0.7$. Contours are as in Fig. 5 .

remove filamentary debris. The figure shows that most erosion of the vortex edge is confined to the upper half of the domain, although the outermost edges in the broad edge case also show some weak erosion even at low levels. The figure also emphasizes the result, already seen in Fig. 9b, that the final area of the upper vortex increases with increasing edge width.

\section{Results with a primitive equation model}

Although the CASL method used above contains the key dynamical features of the winter stratosphere, in particular high horizontal and vertical resolution and a planetary vorticity gradient, it might be argued that quasigeostrophic dynamics might limit the applicability to the real atmosphere. In this section we demonstrate that the edge gradient effects described above are also present in a more realistic primitive equation (PE) model in spherical geometry. In addition to verifying the CASL results, the PE results also point to direct implications for climate modeling in more comprehensive stratospheric GCMs.

We use the "BOB" primitive equation model, described in Rivier et al. (2002), which solves the dry,
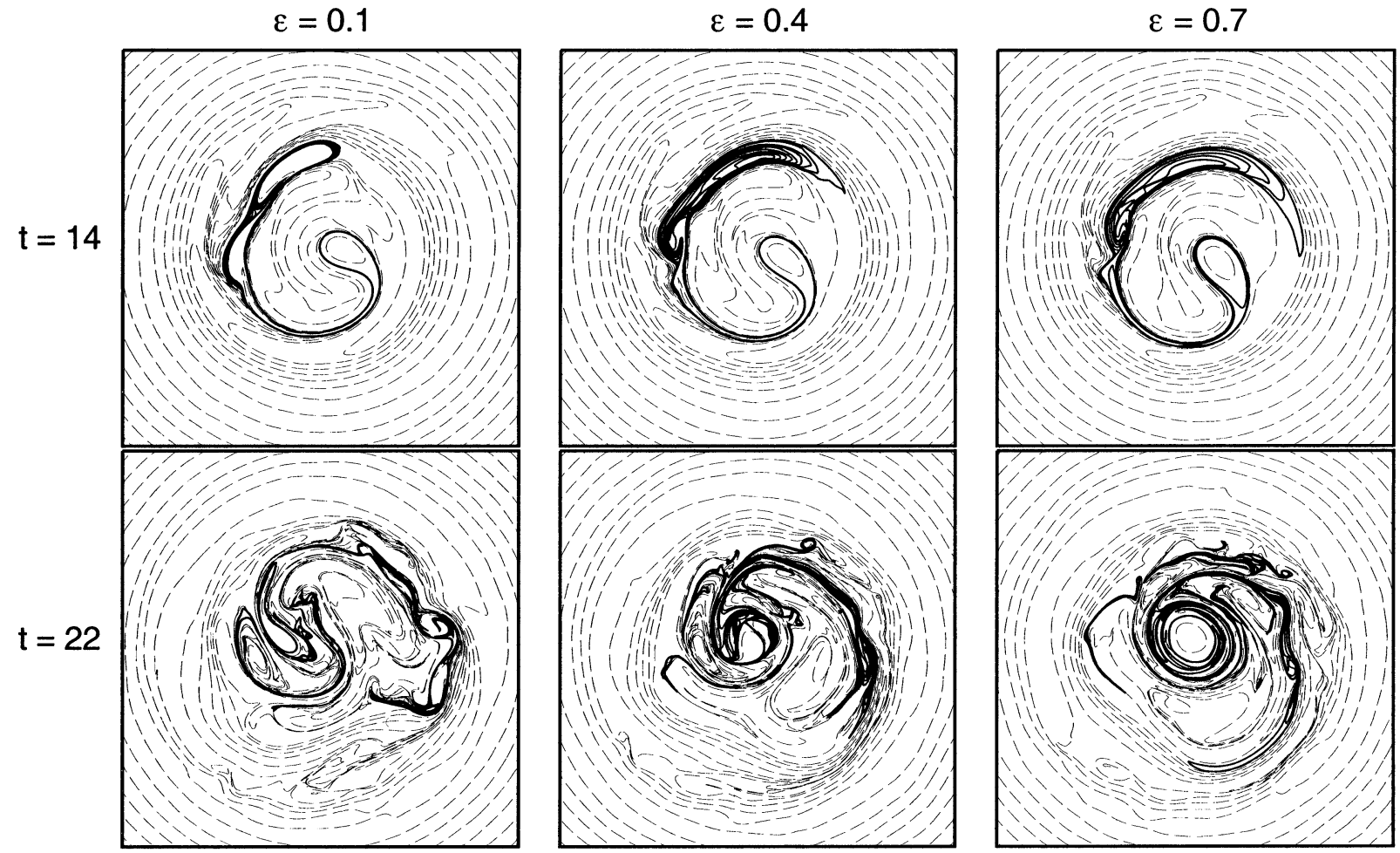

FIG. 8. Potential vorticity of the top model layer, for forcing amplitudes $a=0.26$ at times $t=14$ and $t=22$ days, for edge width $\varepsilon=0.1, \varepsilon=0.4$, and $\varepsilon=0.7$. Contours are as in Fig. 5 . 

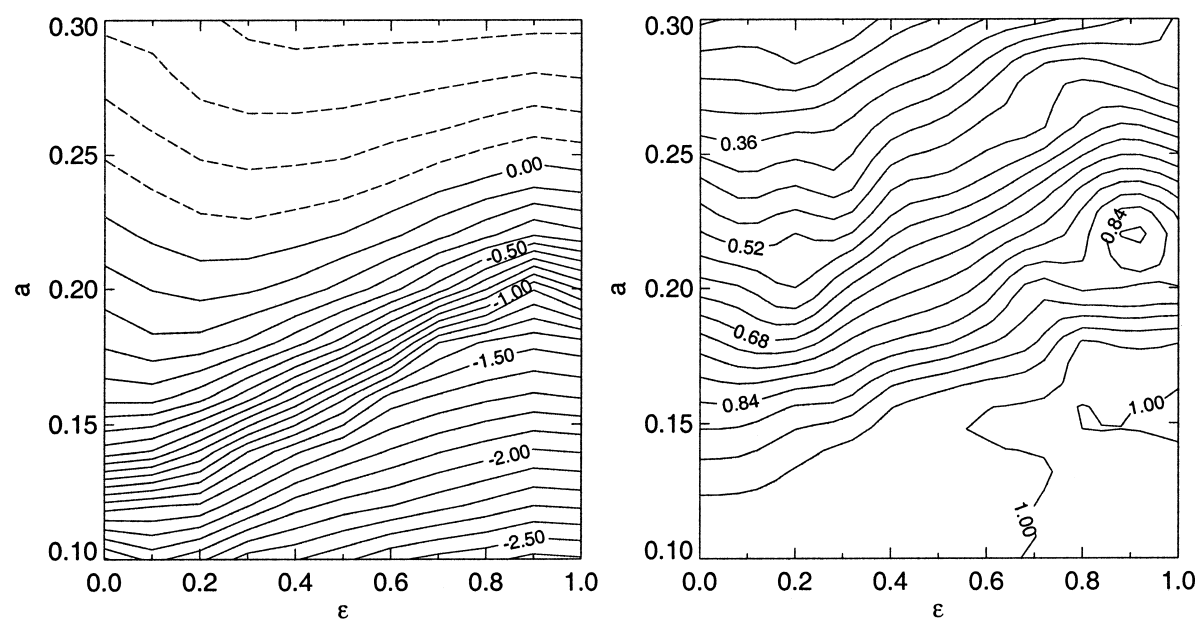

FIG. 9. (a) $\log _{10}$ of wave activity and (b) vortex volume (relative to the initial vortex volume) in the upper $20 \%$ of the vortex averaged from time $t=10$ to $t=20$ days as a function of forcing amplitude $a$ and edge width $\varepsilon$. Both quantities are based on the midedge contour and the volume is calculated after the filamentary debris has been removed by coarse graining.

adiabatic primitive equations in spherical geometry using pressure, $p$ as a vertical coordinate. Horizontal resolutions used are spectral T21, T42, T85, and T170, and there are 40 levels in the vertical, equally spaced in log $p$ between $p_{b}=20000 \mathrm{~Pa}$ and $p_{t}=2 \mathrm{~Pa}$. The model includes a scale-selective $\nabla^{8}$ hyperdiffusion to ensure model stability while minimizing dissipation at the larger scales.

The model is initialized with a barotropic polar vortex of variable edge width having identical properties to that described in section $2 \mathrm{~b}$. The set of different initial conditions is obtained from (2) for the relative vorticity $\zeta$ with the identification $r^{2} \rightarrow a^{2}(1-\sin \phi),(\phi=$ latitude) and the additional requirement that $\zeta$ increases linearly in $a \sin \phi$ from the location of the zero wind line at $30^{\circ} \mathrm{N}$ to the South Pole, where the value is chosen such that the globally integrated PV is zero. The vortex edge ( $R$ above) is chosen to be at $60^{\circ}$ in the case $\varepsilon=$ 0 , and the values of $q_{i}$ and $q_{o}$ are fixed as before, giving a maximum jet of around $55 \mathrm{~m} \mathrm{~s}^{-1}$.

Waves are forced in the lowest model levels, $p_{b}>p$ $>p_{1}=50 \mathrm{hPa}$ by the addition to the potential temperature tendency of a zonal wavenumber-1 heat source $Q$ of the form

$$
\begin{aligned}
& Q(\lambda, \phi, z) \\
& \quad= \begin{cases}A \Phi(\phi) \frac{\log p-\log p_{1}}{\log p_{b}-\log p_{1}} \cos \lambda & p_{1}<p<p_{b} \\
0 & p \leq p_{1}\end{cases}
\end{aligned}
$$

for some constant forcing amplitude $A$, where $\lambda$ is longitude and the latitudinal dependence is given by $\Phi(\pi / 6 \leq \phi<\pi / 2)=\sin ^{2}(1 / 3)(\pi / 2-\phi)$ and $\Phi(\phi<$
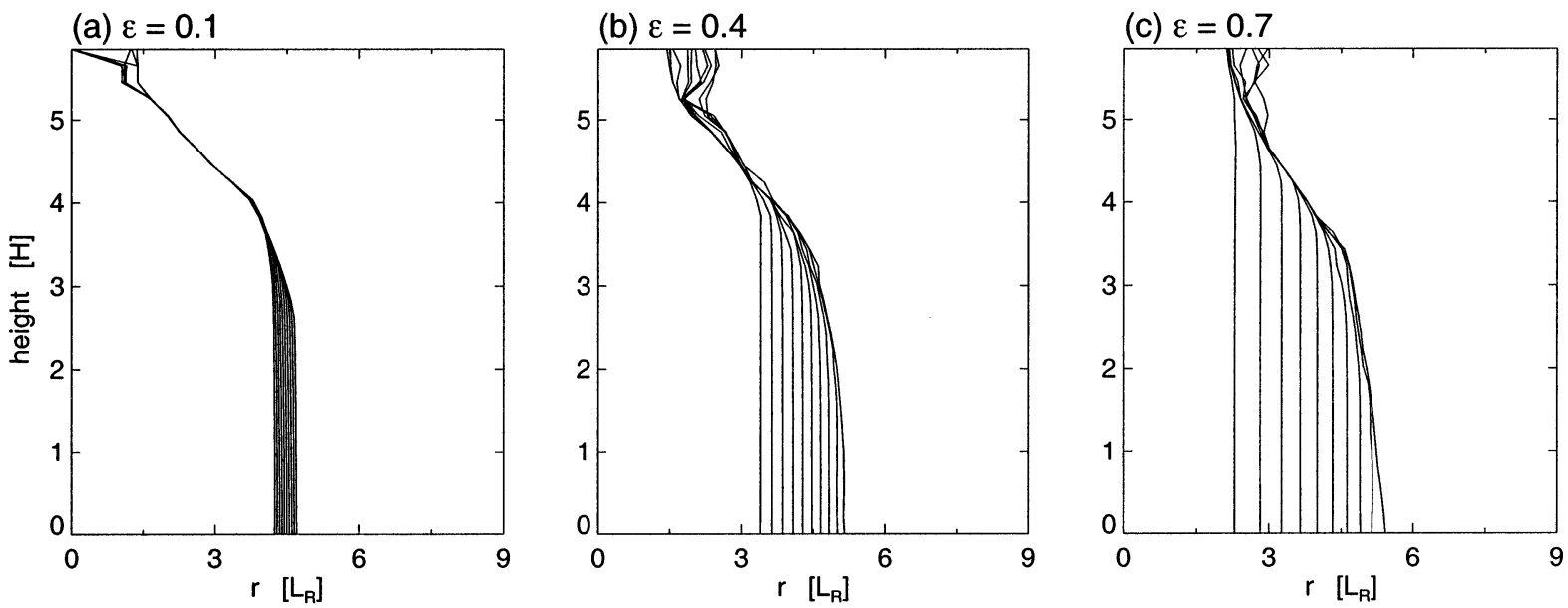

FIG. 10. Equivalent radius of the vortex edge contours as a function of height, for the case (a) $a=0.26$ and $\varepsilon=0.1$, (b) $\varepsilon=0.4$, and (c) $\varepsilon=0.7$, calculated from the coarse-grained PV fields at time $t=30$ days 
(a) $\varepsilon=0.1$

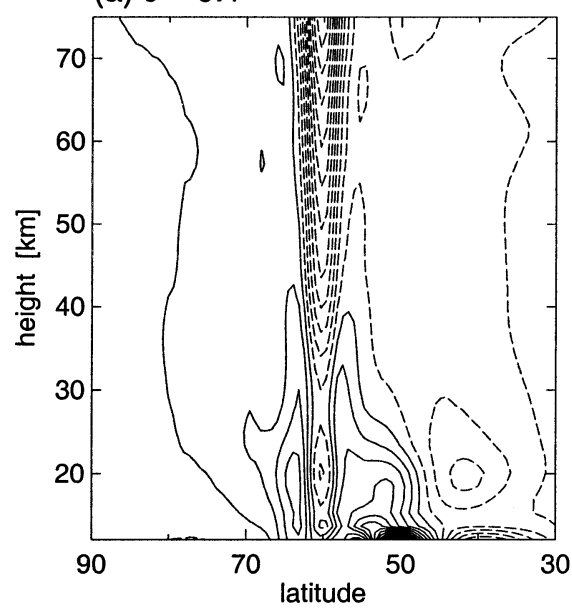

(b) $\varepsilon=0.4$

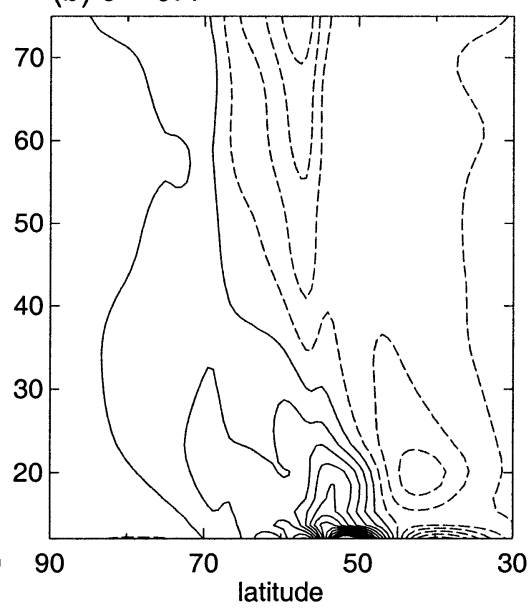

(c) $\varepsilon=0.7$

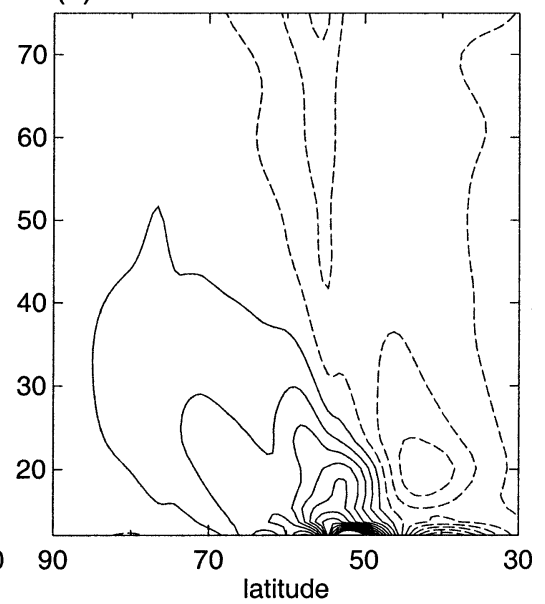

FIG. 11. Deceleration of the vortex in the primitive equation model with a horizontal resolution of spectral T170 after $t=8$ days with thermal wave forcing $A=10^{-4} \mathrm{~K} \mathrm{~s}^{-1}$ in the lowermost stratosphere: (a) $\varepsilon=0.1$, (b) $\varepsilon=0.4$, and (c) $\varepsilon=0.7$. The contour interval is 0.4 $\mathrm{m} \mathrm{s}^{-1}$, and negative contours are dashed.

$\pi / 6)=0$. This wave forcing is applied for the first 10 days of the model integration and then switched off.

We describe two sets of simulations. In the first, we consider high-resolution (spectral T170) simulations with different initial conditions in which the edge width of the barotropic polar vortex is varied systematically with $\varepsilon$. Weak wave forcing is applied with $A=10^{-4} \mathrm{~K}$ $\mathrm{s}^{-1}$, which can be roughly compared to the case $a=$ 0.1 presented in section 4. Figure 11 shows the zonal mean deceleration of the vortex, $\bar{u}(t)-\bar{u}(0)$, as a function of latitude and height at time $t=8$ days, with initial conditions corresponding to the edge widths $\varepsilon=0.1$, $\varepsilon=0.4$, and $\varepsilon=0.7$. As before, we see there is a substantial decrease in the deceleration of the upper vortex for larger $\varepsilon$. That is, even within the resolution restrictions of a spectral PE model, at high enough resolution, differences in the initial edge width can be large enough to cause significant differences in the wave propagation, with less wave propagation on the broader edge. ${ }^{1}$

Note that unlike the CASL simulations described above, the hyperdiffusion used here is necessary to prevent the steepening of vorticity gradients beyond that which can be numerically resolved. At T170, the diffusion coefficient used acts on the smallest resolved scales at a rate of $1440 \mathrm{day}^{-1}$. Thus, even at this relatively high resolution, there is some blurring of the edge by the diffusion, but this is small compared with

\footnotetext{
${ }^{1}$ It should be remembered here that, because of the nature of the tropospheric forcing, the total wave flux into the stratosphere is not independent of $\varepsilon$ in this case. Thus, is it possible that the edge steepness will also affect the magnitude of the total upward EP flux into the stratosphere. Although this effect can be considered to be distinct from the enhancement of upward propagation by small $\varepsilon$, these experiments indicate that the main result of the CASL experiments is robust: namely, that a steep edge results in significantly more wave activity reaching the upper stratosphere.
}

the difference in widths set by the values of $\varepsilon$ shown in the figure. Indeed, examination of a similar series of integrations but with the wave forcing set to zero (not shown), indicates that the deceleration of the vortex due to purely diffusive effects is around an order of magnitude lower than that found in Fig. 11. Thus, at this resolution, differences in deceleration of the vortex are mainly associated with differences in the wave propagation and not with stronger diffusion of the steeper edge. On the other hand, at lower resolutions (T21 or T42), the deceleration of the steep-edged vortex due to diffusion is comparable to that due to wave propagation, and the wave-induced deceleration shows less variation with $\varepsilon$.

The second set of simulations brings into focus some of the implications of this work for low-resolution stratospheric climate modeling. Instead of varying the initial conditions, we fix $\varepsilon=0$, corresponding to a steep edge vortex such as suggested by aircraft observations (Waugh et al. 1994) and perform simulations at varying horizontal resolution. The steep initial condition mimics the attempt of the dynamics to produce steep PV gradients, such as would happen in more realistic forceddissipative calculations. Here, the initial jump is quickly smoothed out by the model diffusion, which is necessarily stronger at lower horizontal resolution.

We consider resolutions of $\mathrm{T} 21, \mathrm{~T} 42, \mathrm{~T} 85$, and $\mathrm{T} 170$, with corresponding diffusion rates of $2,4,24$, and 1440 day $^{-1}$, respectively, at the smallest scales. Stronger wave forcing is applied, with $A=3 \times 10^{-4}$ $\mathrm{K} \mathrm{s}^{-1}$, so that the vortex experiences more pronounced wave breaking. Figure 12 shows the PV on the $1800-\mathrm{K}$ isentrope at time $t=20$ days for each of the four resolutions. The higher PV within the vortex is represented by darker shading, and we have chosen the PV value of $7.5 \times 10^{-4} \mathrm{~K} \mathrm{~kg}^{-1} \mathrm{~m}^{2} \mathrm{~s}^{-1}$ as rep- 
T21

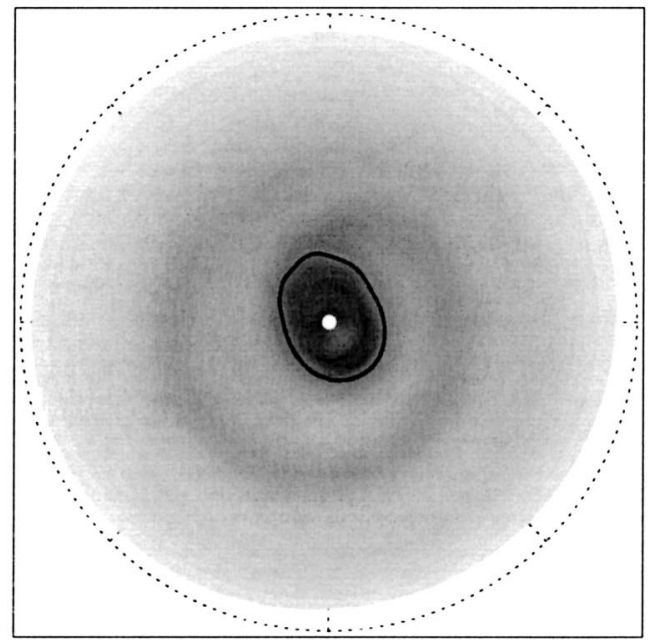

T85

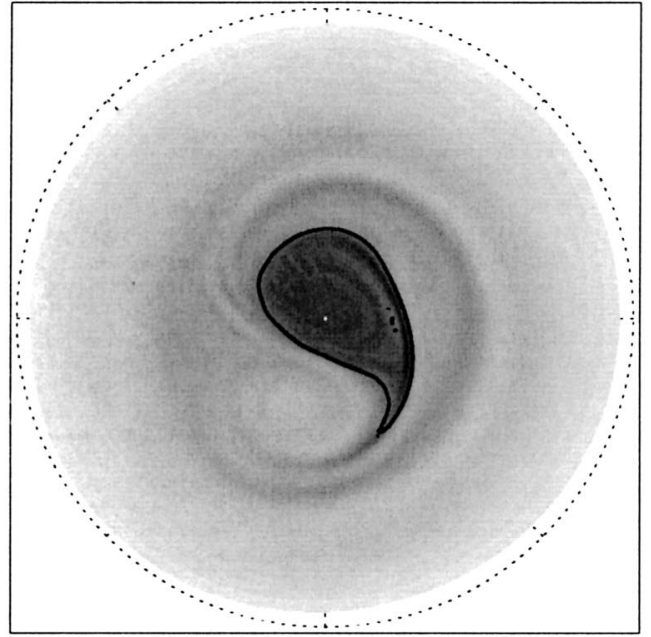

T42

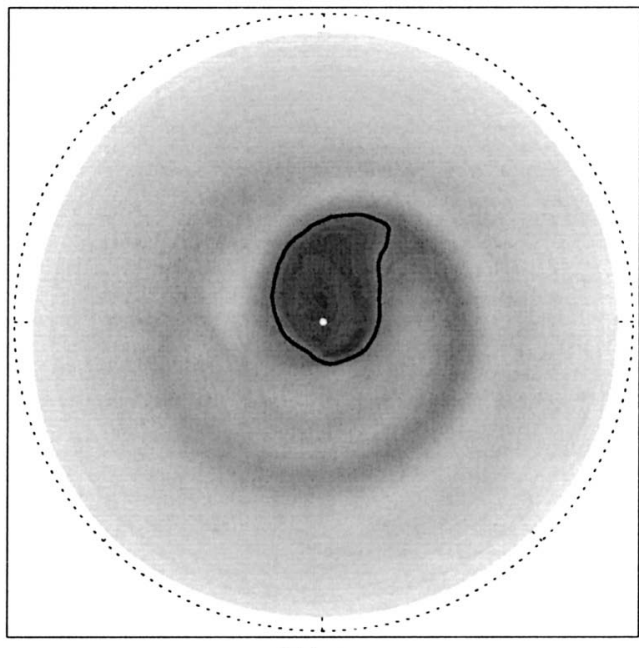

T170

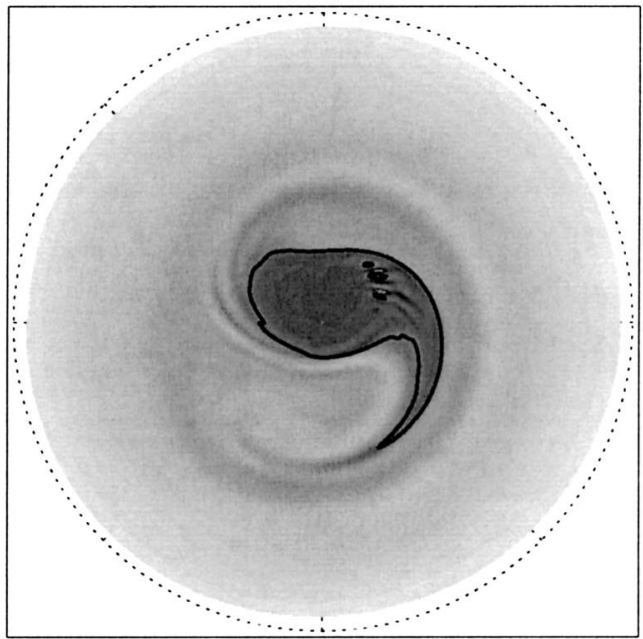

FIG. 12. Potential vorticity at $1800 \mathrm{~K}($ near $45 \mathrm{~km})$ in the primitive equation model after $t=20$ days with thermal wave forcing $A=3 \times 10^{-4} \mathrm{~K} \mathrm{~s}^{-1}$ in the lowermost stratosphere, and initial condition $\varepsilon=0$ for horizontal resolutions of spectral (a) T21, (b) T42, (c) T85, and (d) T170. The solid contour represents a typical vortex edge PV value of $7.5 \times 10^{-4} \mathrm{~K} \mathrm{~kg}^{-1} \mathrm{~m}^{2} \mathrm{~s}^{-1}$.

resentative of the vortex edge (shown in the figure by the solid contour).

At the lowest resolutions, diffusion plays a major role in the reduction of the vortex size and causes a broadening of the width of the vortex edge, as can be seen from the shading. As a result, there is less upward wave propagation and less wave activity at the level shown, and the vortex edge (solid contour) exhibits almost no wave breaking. The filamentation seen at both T21 and T42 arises from erosion (weak wave breaking) of the weak PV gradients on the outer edge region that have been created by the artificial model diffusion.

At higher resolution, on the other hand, diffusion is weaker and the vortex edge is less smeared out, resulting in stronger upward wave propagation and more wave activity reaching the level shown. At T85, a wavenumber-3 distortion of the vortex can be observed as the polar vortex interacts with a smaller anticyclone, and strong wave breaking, involving interior polar vortex air, is associated with this interaction. At T170, a still larger area of polar air is extruded into midlatitudes. The qualitative differences between the low- and highresolution cases is clear: at low resolution, diffusion reduces the vortex size regardless of wave activity and fails to reproduce the mixing of polar air to low latitudes seen at high resolution.

\section{Summary and implications}

We have shown that the steepness of the PV gradients in a vortex directly controls the amount of vertical wave propagation and, consequently, the amount of wave breaking in the upper part of the flow. Although steep PV gradients are traditionally associated with strong 
Rossby elasticity, which acts to suppress wave breaking, wave amplification by the vertical stratification generally causes wave breaking in the upper vortex, regardless of the PV gradients there. The above results indicate that the enhanced wave propagation on steep PV gradients at lower levels has a larger influence on the wave breaking than the elasticity associated with the PV gradients at upper levels. Further, vortex stripping at upper levels tends to intensify PV gradients in the broad edge cases, so that the vortex edge exhibits less dependence on our edge width parameter $\varepsilon$ at upper levels than at low levels (an extreme case is that shown in Fig. 10 after most wave breaking is over, but similar steepening at upper levels is found at earlier times).

Our conclusions hold for both the $f$ plane and $\gamma$ plane, indicating that the effect is due purely to the wave dynamics on the edge, rather than due to the ability of waves on a broader edge to leak to low latitudes on the background PV gradient present on the $\gamma$ plane. Indeed, EP fluxes on the $\gamma$ plane did not indicate any sensitivity to the edge width of the wave propagation to low latitudes.

More significantly, our conclusions also hold for a full primitive equation model in spherical geometry. The CASL method was used for its efficiency in spanning a large parameter space. While we cannot replicate the full parameter sweep at sufficiently high resolution in the PE model, selected cases indicate that the effect is the same in the more realistic model. Further, using the PE model brings into focus some implications of this work for modeling in more comprehensive stratospheric GCMs.

One implication is that forecasts of stratospheric sudden warming events may be hampered by the inability of traditional spectral or gridpoint primitive equation models to represent the steep PV gradients of the stratospheric polar vortex and the associated vertical wave propagation. Even at moderate spectral resolutions this can lead to significant differences in the dynamical evolution, as seen by comparing the T85 and T170 resolutions in Fig. 12.

Perhaps more importantly, on longer time scales stratospheric climate models are restricted to still lower resolution, and the inability to represent steep PV gradients in these models may be a serious deficiency. For example, underestimated wave breaking will lead to a weaker wave-driven mean meridional circulation and a weaker transport of chemical species from the tropical troposphere into the high-latitude winter stratosphere, which will impact ozone chemistry and hence the radiative equilibrium. Similarly, weaker wave breaking and fewer stratospheric sudden warmings will result in less mixing of polar and midlatitude air, again affecting ozone chemistry and polar stratospheric temperatures.

Whether one is interested in short-term forecasts or long climate time scales, the inability to represent wave propagation and breaking accurately will lead to incorrect predictions. In very low resolution models, Fig. 12 indicates another unwanted effect: the horizontal diffusion needed to maintain numerical stability not only prevents the development of sharp PV gradients, but also contributes to the destruction of the polar vortex itself. As has been discussed in previous work in a twodimensional context, the combination of vortex stripping by an external strain flow (or by wave breaking) with large horizontal diffusion, leads to artificially fast reduction in the size of the vortex (Mariotti et al. 1994; Legras et al. 2001; Yao et al. 1995). Essentially, vortex stripping is greatest on a smooth PV distribution, and the diffusion helps to keep the distribution smooth. Hence, the vortices in the T21 and T42 cases in Fig. 12 are smaller than their higher-resolution counterparts, even though wave breaking in these cases is much weaker.

Work is currently underway to address quantitatively the effects of model resolution and diffusion, as well as the effect of radiative forcing, in longer-term simulations of the polar vortex, and to examine the impact on the frequency of sudden warmings, mixing, and transport by the mean meridional circulation.

Acknowledgments. The authors wish to thank an anonymous reviewer for helpful comments and criticism on an earlier version of this manuscript. This work was supported by the U.S. National Science Foundation and by the David and Lucile Packart Foundation. DGD received support for this research from the U.K. Natural Environment Research Council.

\section{REFERENCES}

Andrews, D. G., J. R. Holton, and C. B. Leovy, 1987: Middle Atmosphere Dynamics. Academic Press, 489 pp.

Dritschel, D. G., and R. Saravanan, 1994: Three-dimensional quasigeostrophic contour dynamics, with an application to stratospheric vortex dynamics. Quart. J. Roy. Meteor. Soc., 120, 12671297.

_- and M. H. P. Ambaum, 1997: A contour-advective semi-Lagrangian numerical algorithm for simulating fine-scale conservative dynamical fields. Quart. J. Roy. Meteor. Soc., 123, $1097-$ 1130 .

Dunkerton, T. J., C.-P. F. Hsu, and M. E. McIntyre, 1981: Some Eulerian and Lagrangian diagnostics for a model stratospheric warming. J. Atmos. Sci., 38, 819-843.

Hartmann, D. L., J. M. Wallace, V. Limpasuvan, D. W. J. Thompson, and J. R. Holton, 2000: Can ozone depletion and global warming interact to produce rapid climate change? Proc. Natl. Acad. Sci., 97, 1412-1417.

Hoskins, B. J., M. E. McIntyre, and A. W. Robertson, 1985: On the use and significance of isentropic potential-vorticity maps. Quart. J. Roy. Meteor. Soc., 111, 877-946.

Juckes, M. N., and M. E. McIntyre, 1987: A high resolution, onelayer model of breaking planetary waves in the stratosphere. Nature, 328, 590-596.

Legras, B., and D. G. Dritschel, 1993: Vortex stripping and the generation of high vorticity gradients in two-dimensional flows. Appl. Sci. Res., 51, 445-455.

- and P. Caillol, 2001: The erosion of a distributed twodimensional vortex in a background straining flow. J. Fluid Mech., 441, 369-398.

Macaskill, C., W. E. P. Padden, and D. G. Dritschel, 2003: The CASL 
algorithm for quasi-geostrophic flow in a cylinder. J. Comput. Phys., 188, 232-251.

Mariotti, A., B. Legras, and D. G. Dritschel, 1994: Vortex stripping and the erosion of coherent structures in two-dimensional flows. Phys. Fluids, 6, 3954-3962.

McIntyre, M. E., 1990: Middle atmospheric dynamics and transport: Some current challenges to our understanding. Dynamics, Transport and Photochemistry in the Middle Atmosphere of the Southern Hemisphere: San Francisco NATO Workshop, A. O'Neill, Ed., Kluwer, 1-18.

_ 1992: Atmospheric dynamics: Some fundamentals, with observational implications. Proceedings of the International School of Physics "Enrico Fermi," CXV Course, J. C. Gille and G. Visconti, Eds., North-Holland, 313-386.

Plumb, R. A., and Coauthors, 1994: Intrusions into the lower stratospheric Arctic vortex during the winter of 1991-92. J. Geophys. Res., 99, 1089-1105.

Polvani, L. M., and R. A. Plumb, 1992: Rossby wave breaking, microbreaking, filamentation and secondary vortex formation: The dynamics of a perturbed vortex. J. Atmos. Sci., 49, 462-476.

- and R. Saravanan, 2000: The three-dimensional structure of breaking Rossby waves in the polar wintertime stratosphere. $J$. Atmos. Sci., 57, 3663-3685.

- D. W. Waugh, and R. A. Plumb, 1995: On the subtropical edge of the stratospheric surf zone. J. Atmos. Sci., 52, 1288-1309.
Rivier, L., R. Loft, and L. M. Polvani, 2002: An efficient spectral dynamical core for distributed memory computers. Mon. Wea. Rev., 130, 1384-1390.

Tuck, A. F., 1989: Synoptic and chemical evolution of the Antarctic vortex in late winter and early spring, 1987. J. Geophys. Res. 94, $11687-11737$.

Waugh, D. W., 1992: The efficiency of symmetric vortex merger. Phys. Fluids, 4A, 1745-1758.

— 1993: Contour surgery simulations of a forced polar vortex. $J$. Atmos. Sci., 50, 714-730.

— technique for investigating finescale structure in tracer transport J. Atmos. Sci., 51, 530-540.

— breaking on the vertical structure of the polar vortex. J. Atmos. Sci., 56, 2359-2375.

— - and Coauthors, 1994: Transport of material out of the stratospheric Arctic vortex by Rossby wave breaking. J. Geophys. Res., 99, 1071-1088.

WMO, 1999: Scientific assessment of ozone depletion 1998. WMO Global Ozone Research and Monitoring Project Rep. 44, World Meteorological Organization.

Yao, H.-B., D. G. Dritschel, and N. J. Zabusky, 1995: High gradient phenomena in two-dimensional vortex interactions. Phys. Fluids, 7, 539-548. 